\title{
Cementos belíticos: alternativa ambiental e industrial
}

\section{Belite cements: an environmental and industrial alternative}

Oscar Corona ${ }^{1,3, *}$, Andrea Teixeira ${ }^{2}$, Alejandra Balza ${ }^{3}$

INFORMACIÓN DEL ARTÍCULO

Fecha de recepción: 16 de Febrero de 2018

Fecha de aceptación: 22 de Febrero de 2018
1 Universidad Regional Amazónica Ikiam, Tena, Ecuador.

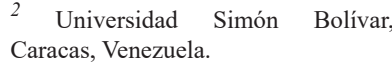

2 Universidad Simón Bolívar, Caracas, Venezuela.

3 PDVSA Intevep, Los Teques, Venezuela.

\footnotetext{
* Autor de correspondencia: Oscar Corona, Universidad Regional Amazónica Ikiam. $\mathrm{Km} 7$ vía Muyuna.

E-mail: oscarcorona78@gmail.com Tlf: (593-6) 3700040.
}

\section{Resumen}

La industria del cemento afronta enormes retos: la alta dependencia de combustibles fósiles para sus procesos de producción, la reducción de las fuentes naturales de materia prima, el aumento exponencial de la demanda y la creciente preocupación por aspectos ambientales. Reportes de la Agencia Internacional de Energía (AIE) resaltan que por cada tonelada de cemento Portland producido se libera a la atmósfera una cantidad similar de $\mathrm{CO}_{2}$, lo que equivale aproximadamente al 6\% de las emisiones antropogénicas. En respuesta a estas problemáticas, se han realizado modificaciones en los esquemas de fabricación del cemento en cuanto al mayor aprovechamiento energético en las plantas de producción y en la búsqueda de soluciones más duraderas y eficientes, se apunta a la incorporación de nanoaditivos para la obtención de cementos belíticos de bajo costo energético con alto desempeño. Sus amplias ventajas son el resultado de la convergencia de múltiples aspectos, basados en la modificación de la química y microestructura de los materiales compuestos.

\section{Palabras Clave:}

Cementos belíticos, cementos verdes, nanomateriales, producción limpia, materiales compuestos.

Clasificación JEL: F18; O13; P28.

\section{Abstract}

Nowadays, the cement industry faces enormous challenges: the high dependence on fossil fuels for its production processes, the reduction of natural raw material sources, the increased demand, and the growing concern about environmental aspects. Reports from the International Energy Agency (IEA) point out that for every ton of Portland cement produced a similar amount of $\mathrm{CO}_{2}$ is released into the atmosphere, which is approximately $6 \%$ of the anthropogenic emissions. In response, some modifications have been made in cement manufacturing for better energy use in production plants, looking for more durable and efficient solutions. The incorporation of nanoadditives is also proposed for the generation of low energy cost and high performance belite cements. Their advantages are the result of the convergence of multiple aspects based on the modification of the chemistry and microstructure of the composite materials.

\section{Keywords:}

Belite cements, green cements, nanomaterials, clean production, composite materials.

JEL Classification: F18; O13; P28. 


\section{Introducción}

Una de las principales actividades económicas a nivel mundial es la explotación de hidrocarburos, la cual satisface las necesidades de energía del mercado internacional. Hoy en día, la industria petrolera se enfrenta a grandes desafíos en la exploración y producción de estos recursos debido a las características de los mismos y de sus yacimientos, requiriéndose procesos no convencionales para garantizar la extracción de los hidrocarburos, e involucrando altas temperaturas $\mathrm{y}$ presiones. Uno de los procesos más empleados es la recuperación secundaria de crudo por métodos térmicos, entre los que destaca la inyección de vapor, la cual ayuda a mejorar la movilidad del fluido hacia la superficie, por mecanismos como expansión térmica de los fluidos del yacimiento, la reducción de la viscosidad del petróleo y su destilación con vapor.

Sin embargo, estos procesos implican temperaturas dependientes de la calidad del vapor, y alrededor de los $200{ }^{\circ} \mathrm{C}$ se produce el deterioro de la integridad de los materiales empleados en los sistemas cementantes para pozos petroleros (Barrios, 2011).

El proceso de cementación consiste en colocar cemento en el espacio anular entre la tubería de revestimiento y la formación del pozo con la finalidad de proveer aislamiento zonal para evitar la migración de fluidos como agua o gas hacia otras zonas del pozo. Sin este aislamiento zonal no es posible alcanzar la máxima producción del pozo por lo que es de suma importancia que el cemento utilizado tenga excelentes propiedades mecánicas y aislantes, es decir, baja permeabilidad y porosidad (Nelson, 1990).

El principal producto de la hidratación del cemento es el gel C-S-H, y ésta es la fase responsable de dar las propiedades mecánicas y microestructurales al cemento. A partir de $110^{\circ} \mathrm{C}$ el cemento hidratado sufre el fenómeno de retrogresión térmica, que es causada por la transformación polimórfica del gel C-S-H hacia una fase llamada alfa silicato dicálcico hidratado $\left(\alpha-\mathrm{C}_{2} \mathrm{SH}\right)$, que es una fase altamente cristalina y expansiva, y genera un deterioro de las propiedades mecánicas del cemento fraguado, haciéndolo más permeable, y por ende afectando el proceso de producción (Scrivener, 2015).

Por estas razones, están siendo aplicadas nuevas tecnologías para la producción de materiales de los sistemas cementantes que resistan estas condiciones y así prolongar la vida útil del pozo, de manera que el proceso de producción no se vea interrumpido.

Por otra parte, la industria cementera es considerada a nivel mundial como una de las más grandes consumidoras de energía y contribuyente significativa en las emisiones de gases invernadero $\left(\mathrm{CO}_{2}\right.$, NOx, etc.). Por tal motivo, es de suma importancia el desarrollo de nuevas tecnologías que involucren el aprovechamiento sustentable de los recursos y de la energía, para así dar cumplimiento con las exigencias ambientales acordadas en el Protocolo de Kioto, aprobado por la ONU en el año 1997. El objetivo principal de este tratado internacional es lograr una disminución 
de las emisiones de gases invernadero a un $5 \%$ menos del nivel de emisiones anteriores. Venezuela se suscribió a dicho protocolo en el 2005, por lo que las industrias deben adecuarse al mismo, y reducir las emisiones por debajo del actual $0,8 \%$ de emisiones antropogénicas de gases invernadero (Figura 1).

Para dar respuesta te a este aspecto ambiental, se han planteado alternativas entorno al rediseño de las plantas de producción de cemento, así como también a la generación de nuevos materiales cementantes verdes o eco-eficientes (Popescu, 2003; Gartner, 2004).

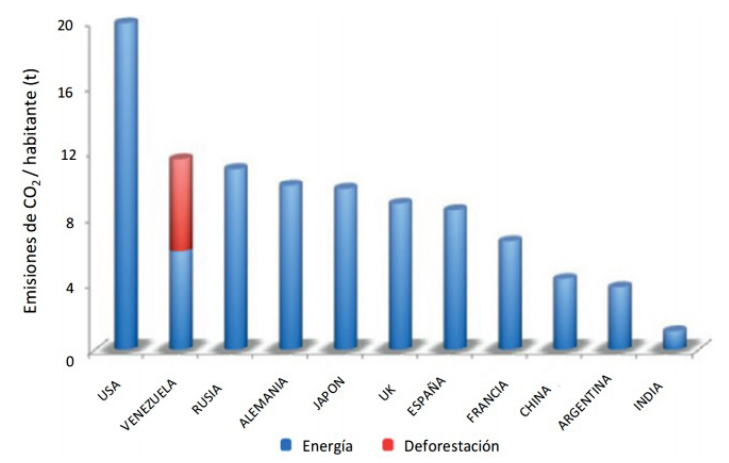

Figura 1. Emisiones de $\mathrm{CO}_{2} /$ habitante en toneladas métricas (2007).

\section{El cemento y su producción}

El cemento es un conglomerante hidráulico, producto de la pulverización del clinker, que cuando es mezclado con agua es capaz de formar una pasta que se endurece mediante reacciones y procesos de hidratación dando lugar a productos hidratados mecánicamente resistentes y estables tanto al aire como sumergidos en agua.

Durante la producción del cemento se combinan químicamente materias primas que abundan en la naturaleza, como las arcillas y las calizas que aportan sílice, alúmina y especies de hierro principalmente. Estos materiales son finamente molidos y calcinados a una temperatura alrededor de $1450{ }^{\circ} \mathrm{C}$, la cual permite una fusión parcial de los mismos para producir un material en forma de solución sólida conocido como clinker, que resulta una mezcla de minerales constituido mayoritariamente por cuatro fases, silicato tricálcico $\left(3 \mathrm{CaO} \cdot \mathrm{SiO}_{2}\right)$, silicato dicálcico $\left(2 \mathrm{CaO} \cdot \mathrm{SiO}_{2}\right)$, aluminato tricálcico $\left(3 \mathrm{CaO} \cdot \mathrm{Al}_{2} \mathrm{O}_{3}\right)$ y ferroaluminato tetracálcico $\left(4 \mathrm{CaO} \bullet \mathrm{Al}_{2} \mathrm{O}_{3} \cdot \mathrm{Fe}_{2} \mathrm{O}_{3}\right)$. Según la notación cementera, dichas fases son conocidas como $\mathrm{C}_{3} \mathrm{~S}, \mathrm{C}_{2} \mathrm{~S}, \mathrm{C}_{3} \mathrm{~A}$ y $\mathrm{C}_{4} \mathrm{AF}$, respectivamente. El clinker es sometido nuevamente a un proceso de molienda en donde se incorpora una cantidad de sulfato de calcio dihidratado $\mathrm{CaSO}_{4} \cdot 2 \mathrm{H}_{2} \mathrm{O}$ (yeso) para controlar la rápida hidratación de la fase aluminato tricálcico e impidiendo el fraguado instantáneo de la mezcla ("flash set") (Taylor, 1997; Hewlett, 1988).

La industria cementera es considerada como un gran consumidor de energía y una de las que más contribuye con la emisión de gases efecto invernadero a nivel mundial. Se estima que corresponde al $5 \%$ del total de las emisiones antropogénicas de $\mathrm{CO}_{2}$. $\mathrm{El} 60 \%$ de la energía total corresponde al proceso de fabricación del clinker en donde sólo el proceso de calcinación del carbonato de calcio $\left(\mathrm{CaCO}_{3}\right)$ absorbe aproximadamente $1,35 \mathrm{GJ} /$ ton de clinker.

Por otro lado, el 50\% de las emisiones de $\mathrm{CO}_{2}$ también están asociadas a la calcinación de los carbonatos, ya que éstos al ser sometidos a elevadas temperaturas desprenden dióxido de carbono al 
ambiente, y el porcentaje restante corresponde a la combustión del carbono presente en los combustibles fósiles empleados para la operación de la planta. Por esta razón, la industria cementera busca optimizar la producción de cemento Portland o reemplazarlo por cementos alternativos de baja emisión de $\mathrm{CO}_{2}$ como cementos con bajo contenido de $\mathrm{C}_{3} \mathrm{~S}$ que requieren menor cantidad de $\mathrm{CaCO}_{3}$ para su producción (Gartner, 2004).

\section{Efecto de las nanopartículas en el cemento}

El objetivo principal de la adición de nanopartículas en cementos es la estimulación de los procesos de nucleación durante la hidratación temprana del cemento. Mientras más temprano esos núcleos son formados, más temprano pueden crecer a grandes cristales de las fases de hidratación y de este modo acelerar la hidratación del cemento, así como también el desarrollo de la resistencia a la compresión y la reducción de la porosidad (Land \& Stephan, 2015).

En la Figura 2 se observa que las nanopartículas actúan como sitios de nucleación y de este modo estimulan que las reacciones hidratación tengan lugar en la superficie de estas partículas. Con la adición de nanopartículas, el espacio entre los granos de cemento es llenado rápidamente con fases de hidratación (Land \& Stephan, 2015).

La naturaleza de los productos de hidratación en mezclas con adiciones de nanopartículas depende de la composición de las mismas. Al incorporar nanosílice se obtiene gel C-S-H con una menor relación $\mathrm{CaO} / \mathrm{SiO}_{2}$, en cambio, con la nanoalúmina se forman geles $\mathrm{C}-\mathrm{A}-\mathrm{S}-\mathrm{H}$ y C-A-H, los cuales se estiman que tienen propiedades parecidas a las del gel C-S-H, debido a que los átomos de aluminio tienen la capacidad de sustituir a los átomos de silicio en la red por tener radios atómicos muy similares. Por lo tanto, estos geles también presentan una menor relación $\mathrm{CaO} / \mathrm{SiO}_{2}$ (Balza, 2016).

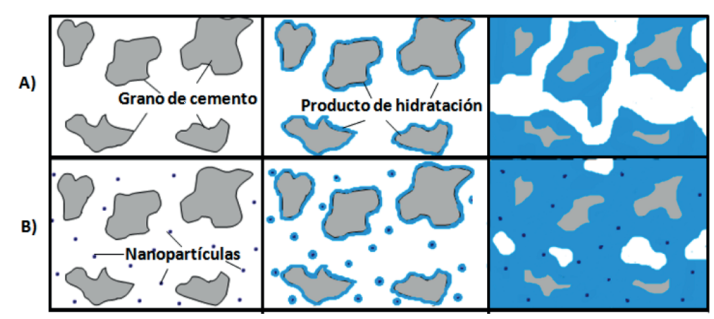

Figura 2. A) Grado de hidratación del cemento puro sin nanopartículas y B) hidratación con nanopartículas, mayor cantidad de productos de hidratación.

\section{Cementos belíticos}

Los cementos belíticos son considerados como "cementos verdes" o "cementos eco-eficientes" ya que, su producción demanda entre 15 y $20 \%$ menos energía que la requerida para la fabricación de un clinker de cemento Portland. Están constituidos mayoritariamente por la fase $\mathrm{C}_{2} \mathrm{~S}$ que implica baja resistencia mecánica inicial por su baja velocidad de hidratación, pero evita problemas de retracción debido a que el calor es liberado de forma progresiva. Además luego de un tiempo desarrolla una mayor resistencia en comparación con el clinker de cemento Portland ordinario (Chartterjee, 1996).

La fase $\mathrm{C}_{2} \mathrm{~S}$ posee cinco formas polimórficas, las cuales son $\alpha, \alpha \mathrm{H}^{\prime}, \alpha \mathrm{L}^{\prime}, \beta$ y $\gamma$ (Tabla 1). Las mismas resultan de 
cambios en la orientación del tetraedro de $\mathrm{SiO}_{4}$ de la forma $\alpha-\mathrm{C}_{2} \mathrm{~S}$ y pequeños movimientos de los iones de calcio, dependiendo de la temperatura. La forma comercial más común en los cementos belíticos es la $\beta-\mathrm{C}_{2} \mathrm{~S}$. La forma $\gamma-\mathrm{C}_{2} \mathrm{~S}$ posee bajas propiedades y es menos densa en comparación con las otras cuatro (Chartterjee, 1996).

Tabla 1.

Formas polimórficas de la fase belita $\left(\mathrm{C}_{2} \mathrm{~S}\right)$ (Chartterjee, 1996).

\begin{tabular}{ccccc}
\hline$\alpha$ & $\alpha^{\prime} \mathrm{H}$ & $\alpha^{\prime} \mathrm{L}$ & $\beta$ & $\gamma$ \\
\hline $1425{ }^{\circ} \mathrm{C}$ & $1160^{\circ} \mathrm{C}$ & $630-680^{\circ} \mathrm{C}$ & $<500^{\circ} \mathrm{C}$ & $780-860^{\circ} \mathrm{C}$ \\
& & & & \\
Hexagonal & Ortorrómbica & Ortorrómbica & Monocíclica Ortorrómbica \\
\hline
\end{tabular}

Existen diferentes métodos que permiten el aumento de la resistencia inicial mediante la estabilización de formas polimórficas reactivas de la belita.

Actualmente, se ha encontrado que dicha estabilización es posible a través de la incorporación de aditivos adecuados, complementado con un enfriamiento rápido. Nuevos métodos también han sido estudiados como "reacción de refusión" o el "método sol-gel", pero su escalamiento industrial resulta un reto (Staněk \& Sulovský, 2015).

Popescu et al. (2002) estudiaron las propiedades mecánicas de cementos belíticos con adiciones de hierro e iones de sulfato y fueron comparadas con el cemento Portland (OPC). Demostraron el potencial que tienen estos cementos como alternativa al Portland, pues evidenciaron que los cementos belíticos tienen una aceptable resistencia a la compresión a los 28 días de hidratación, y que ésta es excelente a edades tardías en donde superan al OPC. Por otra parte, durante la producción del clinker del cemento belítico se consumieron $540 \mathrm{KJ} / \mathrm{Kg}$ menos que para el caso del Portland, asimismo las emisiones de $\mathrm{CO}_{2}$ fueron inferiores (Popescu, 2003).

Producción de los cementos belíticos

El proceso de fabricación del clinker de cemento belítico es el mismo que el del cemento Portland, la diferencia es que para el caso de los cementos belíticos, la materia prima utilizada contiene menor cantidad de carbonato de calcio, la misma es inicialmente molida para lograr un tamaño de partícula alrededor de los $100 \mu \mathrm{m}$. Luego, los materiales de partida son calcinados en un horno rotatorio a una temperatura aproximada de $1200{ }^{\circ} \mathrm{C}$. Finalmente, el producto a la salida del horno es enfriado por diferentes métodos dependiendo de las características finales que se deseen conseguir en el clinker belítico (Popescu, 2003).

Kacimi et al. (2009) reportan que un rápido enfriamiento mediante templado en agua mejora las propiedades hidráulicas del cemento con la estabilización de la estructura reactiva de la belita $\left(\beta-\mathrm{C}_{2} \mathrm{~S}\right)$ a temperatura ambiente, como se evidencia en la Tabla 2.

Tabla 2.

Resistencia mecánica del clinker de cemento belítico (Kacimi, 2009).

\begin{tabular}{|c|c|c|c|c|}
\hline \multirow{2}{*}{$\begin{array}{l}\text { Clinker } \\
\text { belítico }\end{array}$} & \multirow{2}{*}{$\begin{array}{c}\text { Modo de } \\
\text { enfriamiento }\end{array}$} & \multicolumn{3}{|c|}{ Resistencia a la compresión } \\
\hline & & 2 días & 7 días & 28 días \\
\hline \multirow{3}{*}{ Ordinario } & Al aire & 1,43 & 4,17 & 15,97 \\
\hline & & & & \\
\hline & Templado al agua & 6,26 & 16,57 & 34,78 \\
\hline
\end{tabular}

Por otra parte, Guerrero et al. (2004, 2005), consideran que el método más eco-eficiente 
para la producción de cementos belíticos es la ruta de calcinación hidrotérmica utilizando como materia prima residuos industriales como ceniza volante proveniente de la combustión del carbón. En primer lugar, la ceniza volante junto con agua desionizada o solución alcalina es activada hidrotérmicamente a una temperatura alrededor de los $200^{\circ} \mathrm{C}$, a una presión de 1,24 MPa por 4 horas continuas. Durante este proceso, la reacción puzolánica de la ceniza volante es fuertemente activada llevando a productos hidráulicos. Luego, el sólido es secado a $80{ }^{\circ} \mathrm{C}$ y calcinado a $800{ }^{\circ} \mathrm{C}$. De este modo, es posible obtener mayor cantidad de la fase $\mathrm{C}_{2} \mathrm{~S}$ libre de otras fases no deseadas como la gehlenita y cal libre, las cuales tienen baja actividad hidráulica y además producen expansión durante la hidratación (Figura 3) (Guerrero, 2004; Guerrero, 2005).
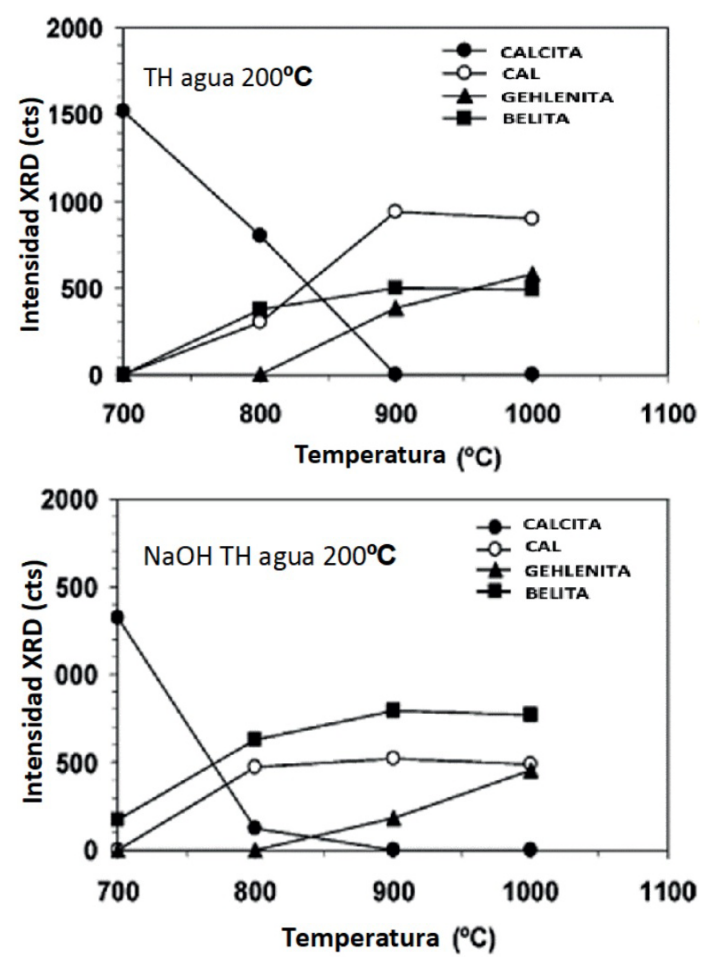

Figuras 3. Evolución semicuantitativa de las fases cristalinas obtenidas a partir de la calcinación de los precursores obtenidos por tratamiento hidrotérmico (TH) en agua y en $\mathrm{NaOH}$. (Guerrero, 2004).
Cementos belíticos y nanotecnología

El estudio de cementos belíticos aditivados con nanomateriales ha sido realizado por diversos investigadores utilizando distintos materiales nanoparticulados. A continuación se muestran algunos de estos estudios junto con los resultados obtenidos en los mismos.

Campillo et al. (2007) estudiaron el potencial que tienen los nanomateriales para la activación de la resistencia inicial de los cementos belíticos. Para esto, utilizaron un cemento belítico sintetizado por la ruta de calcinación hidrotermal y usando ceniza volante como materia prima. Incorporaron dos tipos de nanoalúmina, la primera es seca con un tamaño de grano de $100 \mathrm{~nm}$ y la segunda es alúmina coloidal. Los morteros se prepararon con arena y una relación agua/cemento de 0,8 con adiciones de 0 , 3 y $9 \%$ en peso de cemento de los dos tipos de nanoalúmina. Las muestras se dejaron a $21{ }^{\circ} \mathrm{C}$ y $90 \%$ de humedad relativa por 7 y 28 días. En cada período de tiempo midieron la resistencia a la compresión, porosidad y aplicaron difracción de rayos $\mathrm{X}$, cuyos resultados les permitió concluir que la nanoalúmina puede ser utilizada como un agente reactivo para incrementar la actividad hidráulica de la fase belita presente en estos cementos y también refina la microestructura de la pasta conduciendo al mejoramiento de la resistencia mecánica a edades tempranas (Campillo, 2007).

Dolado et al. (2007) estudiaron el efecto de las adiciones de nanosílice en pastas de 
cementos belíticos curadas en soluciones con sulfatos. Para esto, sintetizaron un cemento belítico por la ruta de calcinación hidrotermal y usando ceniza volante como materia prima. Se prepararon dos pastas con una relación agua/cemento de 0,75 , una consistió en la referencia y a la otra se le incorporaron $7,5 \%$ en peso de cemento de nanosílice coloidal. Las muestras fueron desmoldadas luego de dos días y se colocaron en una solución de sulfato de sodio $0,5 \mathrm{M}$ por 180 días. Se realizaron ensayos mecánicos, difracción de rayos $\mathrm{X}$ y mediciones de ${ }^{29} \mathrm{Si}$ MAS-NMR. L o s resultados arrojaron que la adición de nanosílice induce inicialmente un decaimiento en la resistencia a la compresión de las muestras, solo aquellas mantenidas a 180 días presentaron un aumento de la resistencia. Con la resonancia magnética nuclear se observó que la polimerización del gel C-S-H es más rápida en presencia de nanosílice pero que la longitud de cadena de este gel solo aumenta a los 180 días para las pastas con aditivo. También se encontró la formación de partículas de sílice, a los 7 y 28 días en las muestras aditivadas, que competían con el gel C-S-H por el silicio, lo que explica la rápida polimerización debido a que las cadenas de silicato eran cortas (Dolado, 2007)

\section{Perspectivas y conclusiones}

El estudio del efecto de la temperatura sobre los cementos belíticos aditivados con nanomateriales y su aplicación en sistemas cementantes para pozos petroleros está siendo desarrollado actualmente, por lo que es una investigación de carácter innovador. La ventaja de evaluar los cementos belíticos reside en que para su fabricación se necesita una energía considerablemente menor que para la producción de los cementos utilizados actualmente en la industria petrolera, lo que implica un menor costo tanto económico como ambiental, que hoy en día es un tema de vital importancia. Además, debido a que durante su hidratación los cementos belíticos generan menor cantidad de portlandita y muestran mayor durabilidad en ambientes agresivos (altamente ácidos o alcalinos), lo que permite economizar en aditivos. Por otra parte, su trabajabilidad no es un problema mayor debido a la baja velocidad de hidratación de la belita, lo que a su vez mitiga problemas de retracción.

Con la adición de nanopartículas de alúmina y sílice, se consigue acelerar la velocidad de hidratación para lograr un incremento en la resistencia a la compresión en períodos más cortos, así como también otorgar al sistema resistencia a elevadas temperaturas.

\section{Referencias}

Balza, A., Corona, O., Alarcón, A., Echevarrieta, J., Goite, M., \& González, G. (2016). Estudio Microestructural del Cemento Portland aditivado con Nanomateriales. Acta Microscópica, 25, 39-47.

Barrios, M. J. (2011). Tesis de pregrado: Evaluación de la factibilidad de implementación de la inyección alternada de vapor de agua con gas en la faja petrolifera del Orinoco mediante el uso de un simulador numérico. Universidad de Oriente, Barcelona- Venezuela.

Campillo, I., Guerrero, A., Dolado, J. S., Porro, A., Ibánez, J. A., \& Goñi, S. (2007). Improvement of initial mechanical stregth by nanoalumina in belite cements. Materials Letters, 61, 1889-1892. 
Chartterjee, A. K. (1996). High belite cements-present status and future technological options: part I. Cement and concrete research, 26 (8), 1213-1225.

Dolado, J. S., Campillo, I., Erkizia, E., Ibánez, J. A., Porro, A., Guerrero, A., \& Goñi, S. (2007). Effect of Nanosilica Additions on Belite Cement Pastes Held in Sulfate Solutions. Journal of the American Ceramic Society, 90, 3973-3976.

Guerrero, A., Goñi, S., Campillo, I., \& Moragues, A. (2004). Belite Cement Clinker from Coal Fly Ash of High Ca Content. Optimization of Synthesis Parameters. Environmental science \& technology, 38, 3209-3213.

Guerrero, A., Goñi, S., Moragues, A., \& Dolado, J. S. (2005). Microstructure and Mechanical Performance of Belite Cements from High Calcium Coal Fly Ash. Journal of the American Ceramic Society, 88 (7) 1845-1853.

Hewlett, P. (1era Edición) (1988). Lea's Chemistry of Cement and Concrete. Burlington: Elsevier Ltd.

Kacimi, L., Simon-Masseron, A., Salem, S., Ghomari, A., \& Derriche, Z. (2009). Synthesis of belite cement clinker of high hydraulic reactivity. Cement and Concrete Research, 39, 559-565.

Land, G., \& Stephan, D. (2015). Controlling cement hydration with nanoparticles. Cement and concrete research, 57, 64-67.

Lander, L. E. (2004). La insurrección de los gerentes: PDVSA y el gobierno de Chávez. Revista Venezolana de Economía y Ciencias Sociales, 10(2), 13-32.

Nelson, E. B. (1era Edición) (1990). Well Cementing. Amsterdam: ELSEVIER.

Popescu, C. D., Muntean, M., \& Sharp, J. H. (2003). Industrial trial production of low energy belite cement. Cement and Concrete Composites, 25, 689-693.
Scrivener, K. L., Juilland, P., \& Monteiro, P. J. (2015). Advances in understanding hydration of Portland cement. Cement and Concrete Research, 78, 38-56

Staněk, T., \& Sulovský, P. (2015). Active low-energy belite cement. Cement and Concrete Research, 68, 203-210.

Taylor, H. F. (1era Edición) (1997). Cement Chemistry. Londres: Thomas Telford. 